\title{
Structural Investigation of the pre-40S Ribosomal Particle Using Electron Cryomicroscopy
}

Cherisse Loucks ${ }^{1,3}$, Min $\mathrm{Su}^{1,3}$, Bethany Strunk ${ }^{2}$, Justin Schilling ${ }^{3}$, Katrin Karbstein ${ }^{1,2}$, and Georgios Skiniotis ${ }^{1,3}$

${ }^{1}$ Life Sciences Institute, University of Michigan, 210 Washtenaw Avenue, Ann Arbor, MI 48109-2216

Departments of ${ }^{2}$ Biological Chemistry and ${ }^{3}$ Chemical Biology, University of Michigan, 210 Washtenaw Avenue, Ann Arbor, MI 48109-2216

Mature eukaryotic ribosomes are composed of 4 rRNAs and approximately 80 proteins. The maturation process involves a large number of non-ribosomal proteins that perform specific modifications, such as protein phosphorylation and rRNA cleavage. The process begins in the nucleolus and proceeds with export into the nucleus and cytoplasm upon maturation events. Both the mature $40 \mathrm{~S}$ and $60 \mathrm{~S}$ subunits of the ribosome are generated from their precursors, pre-40S and pre-60S. The precursor particles contain ribosomal proteins and rRNAs, as well as the non-ribosomal proteins that are transiently associated with the complex at different stages of the maturation process.

The identity of the non-ribosomal proteins is well investigated but their location within the complex at different points in maturation is not known. Earlier cryo-EM studies have suggested that despite an overall similarity, there are differences between the structures of the pre-40S and mature 40S complexes [1]. We employ molecular electron microscopy techniques to characterize the structure of the Saccharomyces cerevisiae pre-40S complex, which is known to include at least 8 non-ribosomal proteins and an immature 3' extension on the rRNA. To this end, we are using cryo-EM to produce $3 \mathrm{D}$ reconstructions of pre-40S complexes. Additionally, we use antibody labeling and negative stain EM to determine the localization of non-ribosomal proteins within the complex.

Our 3D cryo-EM maps of the pre-40S ribosomal complex display densities not present in the yeast mature 40S structure [2]. The antibody-labeling studies confirm that some of these areas can be attributed to the presence of non-ribosomal proteins known to associate with premature particles. We are working on the calculation of higher resolution maps to confirm the location of each non-ribosomal protein, trace the path of the immature rRNA, and determine the interactions between the rRNA and proteins within the complex. Ultimately these structural studies will be used to delineate the mechanistic process of ribosome biogenesis.

[1] Thorsten Schäfer et al., Nature. 441 (2006) 651.

[2] D.J. Taylor et al., Structure. 17 (2009) 1591. 


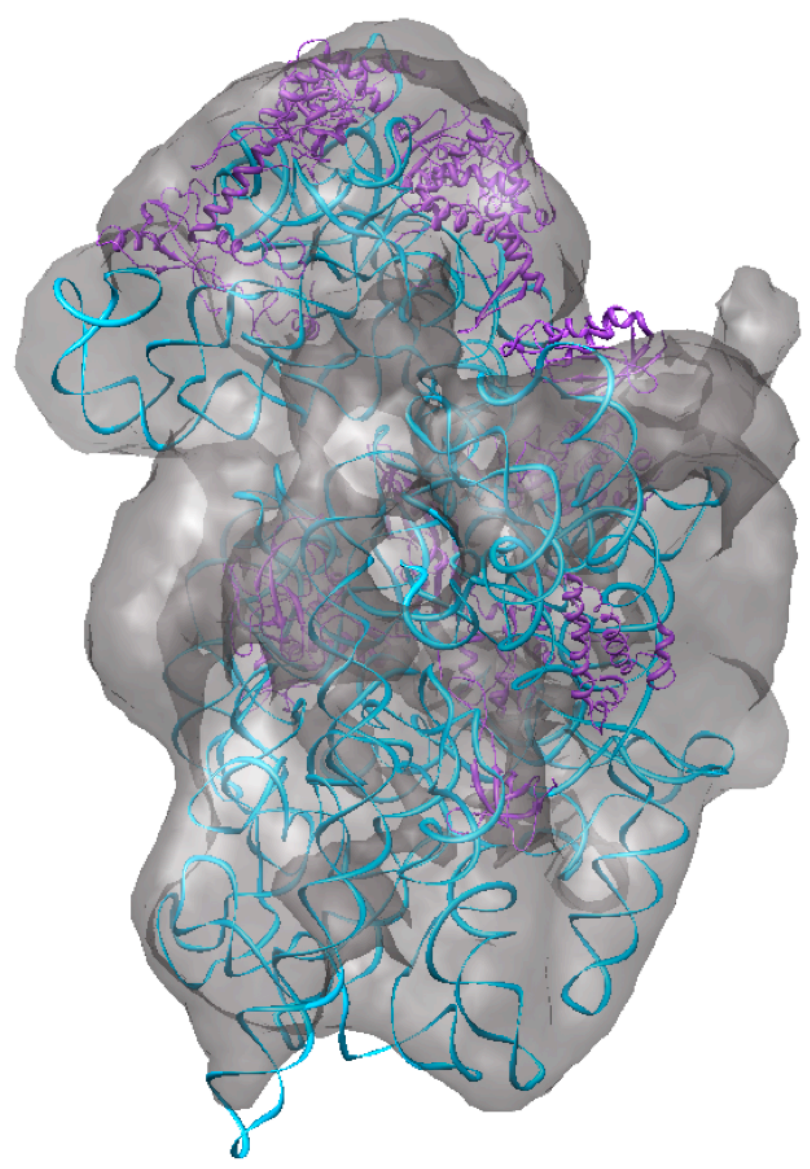

Figure 1: Preliminary cryo-EM 3D volume of Saccharomyces cerevsisae pre-40S with mature 40S structure docked (PDB: 3JYV) [2]
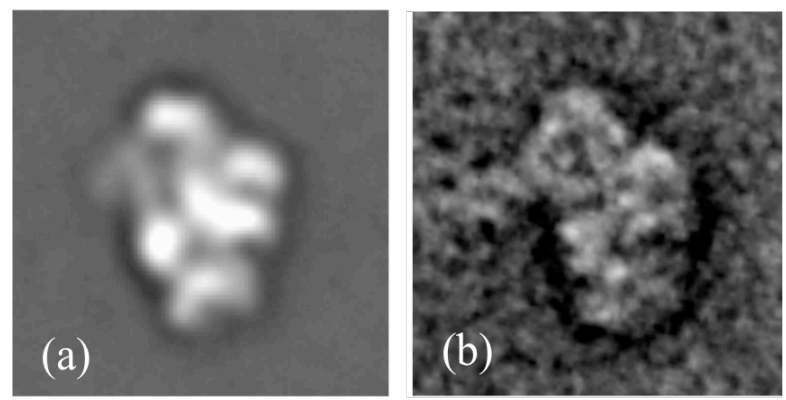

Figure 2: (a) Class averages of pre-40S particle after negative staining, (b) Class averages of antibody labeling of known non-ribosomal protein 